\title{
High-Performance Pseudocubic Thermoelectric Materials from Non-cubic Chalcopyrite Compounds
}

\author{
Jiawei Zhang, Ruiheng Liu, Nian Cheng, Yubo Zhang, Jihui Yang, Ctirad Uher, Xun Shi,* \\ Lidong Chen, * and Wenqing Zhang*
}

Energy harvesting requires clean and highly efficient energyconversion technologies. Thermoelectricity (TE) is one such technology that achieves thermal-to-electric conversion, and vice versa, by purely solid-state means and has a great potential for applications in waste-heat recovery, air conditioning, and spot cooling of electronic devices, by the virtue of environmental friendliness, the dispensing of moving parts, quiet operation, and exceptional reliability. ${ }^{[1-3]}$ In recent years, major effort world-wide has been directed towards the optimization of current TE materials or the exploration of novel high-performance compounds. ${ }^{[4-11]}$ The performance of a TE material is characterized by its dimensionless figure of merit, $z T=S^{2} \sigma T / \kappa$, where $S$ is the Seebeck coefficient, $\sigma$ is the electrical conductivity, $\kappa$ is the thermal conductivity, and $T$ is the absolute temperature. To enhance $z T$ requires a simultaneous realization of excellent electronic transport properties and poor heat conduction in solid materials. The state-of-the-art materials with relatively high $z T$ are limited to only a few semiconductors, most of them narrow-gap structures developed in the $1960 \mathrm{~s} .{ }^{[3]}$

Current TE materials usually possess high-symmetry crystal structures that support the presence of highly degenerate, multi-valley electronic bands yielding good electronic properties characterized by a large power factor $S^{2} \sigma \cdot{ }^{[2,3]}$ As a consequence, most of the state-of-the-art TE materials have cubic structures with degenerate band edges and symmetry-related multi-valley carrier pockets. Typical examples are SiGe alloys, ${ }^{[1,3]} \mathrm{PbTe},{ }^{[1-3]}$ skutterudites, ${ }^{[8]}$ half-Heusler alloys, ${ }^{[12]} \mathrm{Mg}_{2} \mathrm{Si}^{[13]}$ and liquid-like

J. Zhang, Dr. R. Liu, N. Cheng, Dr. Y. Zhang, Prof. X. Shi, Prof. L. Chen, Prof. W. Zhang State Key Laboratory of High Performance Ceramics and Superfine Microstructure Shanghai Institute of Ceramics

Chinese Academy of Sciences, 1295 Dingxi Road Shanghai 200050, China

E-mail:xshi@mail.sic.ac.cn; cld@mail.sic.ac.cn; wqzhang@mail.sic.ac.cn

J. Zhang, N. Cheng

University of Chinese Academy of Sciences

Beijing 100049, China

Prof. J. Yang

Materials Science and Engineering Department

University of Washington

Seattle, Washington 98195-2120, USA

Prof. C. Uher

Department of Physics, University of Michigan

Ann Arbor, Michigan 48109, USA

DOI: 10.1002/adma.201400058
$\mathrm{Cu}_{2}$ Se. ${ }^{[5]}$ A notable exception is $\mathrm{Bi}_{2} \mathrm{Te}_{3}{ }^{[2,3]}$ and compounds based on it that have comparatively high hexagonal symmetry. This severely restricts the exploration of TE materials to a small percentage of semiconductors that possess high-symmetry cubic structures, and thus excludes a large number of low-symmetry non-cubic materials even though they might manifest ideal bandgaps and low thermal conductivities. Directly determined by the chemical nature of its constituents, the crystal structure of a given material is rigid and rarely can be turned from low symmetry to high symmetry short of external stimuli such as pressure. It remains a key challenge to discover or design novel high-performance TE compounds among non-cubic materials. In this work, taking a hint from the recently emerging chalcopyrite TE materials with reasonable $z T$ values ${ }^{[14-22]}$ we report on our successful approach of rationally tuning crystal structures to design pseudocubic or cubic-like structure blocks in non-cubic materials that lead directly to cubic-like degenerate band-edge electronic states and thus high power factors and enhanced $z T$ values in a few carefully selected chalcopyrites (see Figure 1).

The pseudocubic structure approach is here understood as a realization of cubic-like, highly degenerate electronic bands at band edges of non-cubic materials through a complex architecture containing an inherently long-range, nearly cubic framework as well as localized short-range non-cubic lattice distortions (see Figure 1b). Electronic transport processes are dominated by the long-range cubic framework displaying cubic-like highly degenerate band edges and prospects for multi-valley carrier pockets, while the heat conduction is blocked by the presence of large, locally non-cubic lattice distortions. Thus, a very special character of the pseudocubic structure allows the design of high-performance novel TE materials with the ability to simultaneously optimize electrical and thermal transport properties. Binary zinc blende materials have a typical cubic structure with degenerate electron band edges (see Figure 1a). Starting from non-cubic tetragonal chalcopyrites, the cation sublattice can be tuned to show cubic or nearly cubic framework, while the anion sublattice shows a locally distorted non-cubic framework with two types of irregular tetrahedra in ternary chalcopyrites, leading to a periodic supercell with a cubic framework (Figure 1b). The high symmetry cubic supercell results in cubic-like degenerate electron bands at the gamma point of the folded Brillouin zone in tetragonal chalcopyrites, representing an ideal pseudocubic structure (see Figure 1b). Furthermore, through a rationally designed mixing strategy, the emerging complex solid solution chalcopyrites might show an increased randomness of the locally irregular tetrahedra while maintaining the cubic-like 

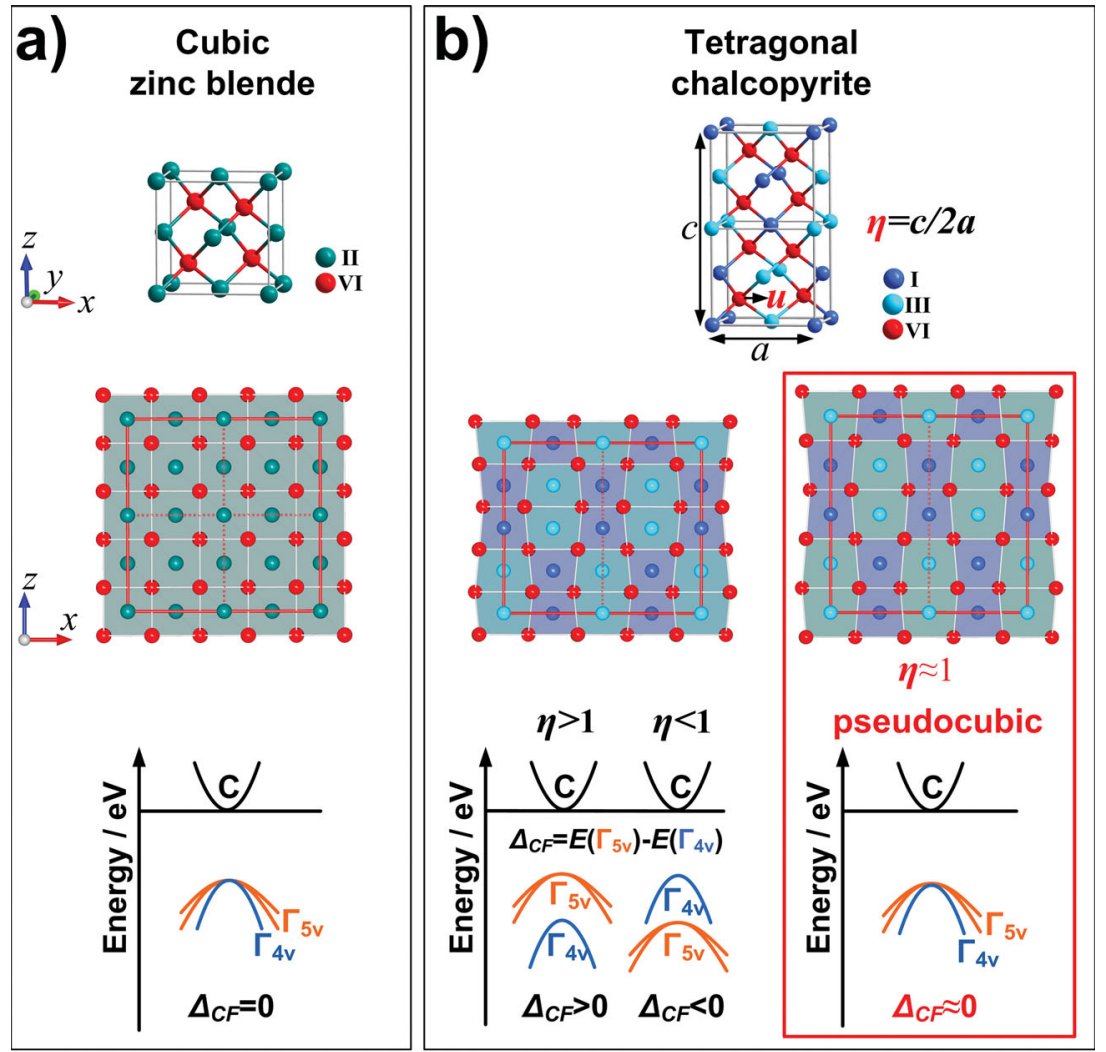

c)

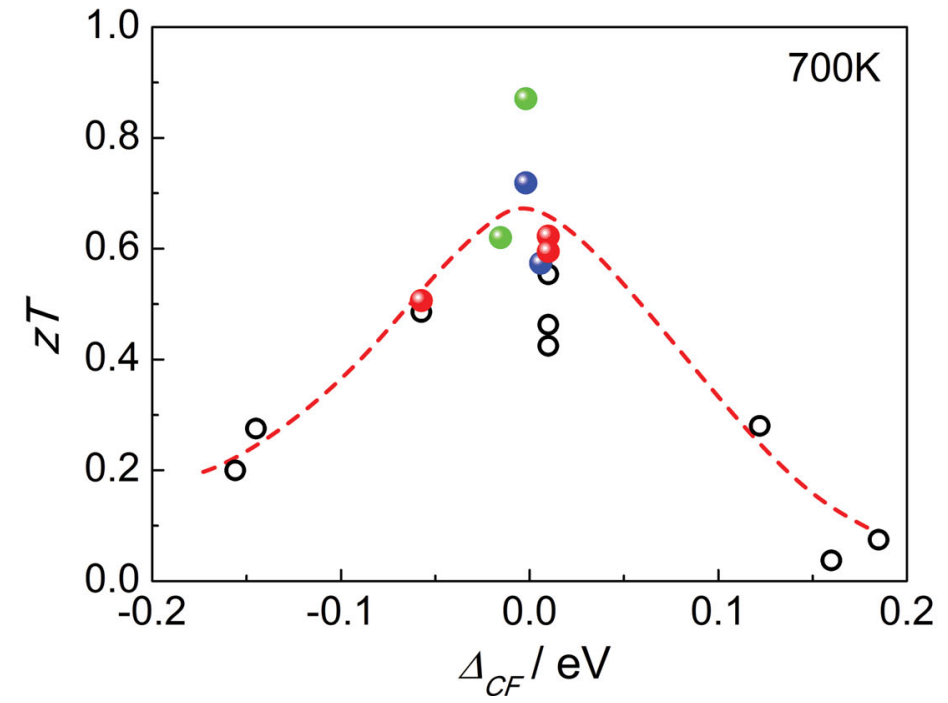

Figure 1. Pseudocubic approach to realize highly degenerate electronic bands that yield good electronic-transport properties and a high thermoelectric figure of merit $z T$ in non-cubic chalcopyrites. a) Crystal structure and electronic bands of cubic zinc blende structure. b) Crystal structure and electronic bands of ternary chalcopyrites. $\Gamma_{4 \mathrm{v}}$ is a nondegenerate band and $\Gamma_{5 \mathrm{v}}$ is a doubly degenerate band. $\Delta_{\mathrm{CF}}$ is the energy split at the top of $\Gamma_{4 \mathrm{v}}$ and $\Gamma_{5 \mathrm{v}}$ bands. The pseudocubic structure has a cubic cation framework with $\Delta_{\mathrm{CF}} \approx 0$ as well as non-cubic distorted anion framework. c) $z T$ values ${ }^{[14-22]}$ at $700 \mathrm{~K}$ in non-cubic tetragonal chalcopyrites with calculated $\Delta_{\mathrm{CF}}$ values. Reported chalcopyrite compounds with $z T$ values ${ }^{[14,17]}$ above unity at high temperature are marked in red. The blue and green points represent $(\mathrm{Ag}, \mathrm{Cu}) \ln \mathrm{Te}_{2}$ (this work, see below for details) and $\mathrm{Cu}(\mathrm{In}, \mathrm{Ga}) \mathrm{Te}_{2}$ (ref. $\left.{ }^{[22]}\right)$ mixtures or solutions, respectively. degenerate electronic states at band edge. Both features bode well for achieving high TE performance with the $z T$ values much enhanced (see below for details) in noncubic tetragonal chalcopyrites ${ }^{[14-22]}$ as shown in Figure 1c and Figure S10 in the Supporting Information.

Chalcopyrite compounds with multiple elements in the crystal lattice analogous to the cubic zinc blende structure cover a wide range of compositions with semiconducting properties. Chalcopyrites usually possess non-cubic tetragonal structures with distorted tetrahedra. They have a doubled unit cell in the $z$-direction (Figure 1b) due to the ordered arrangement of different types of cations. ${ }^{[23]}$ In contrast to the zinc blende lattice, the triply degenerate valence band $\Gamma_{15 \mathrm{v}}$ in tetragonal chalcopyrites splits, due to the crystal field effect, ${ }^{[23,24]}$ into a non-degenerate band $\Gamma_{4 \mathrm{v}}$ and a doubly degenerate band $\Gamma_{5 \mathrm{v}}$ (Figure S1, Supporting Information). The energy difference between $\Gamma_{5 \mathrm{v}}$ and $\Gamma_{4 \mathrm{v}}$ is defined as the crystal field splitting energy, $\Delta_{\mathrm{CF}}=E\left(\Gamma_{5 \mathrm{v}}\right)-E\left(\Gamma_{4 \mathrm{v}}\right)$, which is positive when $\Gamma_{5 \mathrm{v}}$ lies above $\Gamma_{4 \mathrm{v}}$ and negative when $\Gamma_{5 \mathrm{v}}$ lies below $\Gamma_{4 \mathrm{v}}$. The absolute value of $\Delta_{\mathrm{CF}}\left(\left|\Delta_{\mathrm{CF}}\right|\right)$ could thus be considered as a parameter indicating the deviation from pseudocubic or cubic-like degenerate electronic bands of tetragonal chalcopyrite compounds. The degree of deviation decreases as $\left|\Delta_{\mathrm{CF}}\right|$ becomes smaller, attaining a pseudocubic structure with a triply degenerate valence band when $\left|\Delta_{\mathrm{CF}}\right|$ equals zero (shown in the top panel of Figure S1b in the Supporting Information). Figure $\mathrm{S} 1$ also shows the calculated band structures of two types of chalcopyrites, one subjected to a compression stress and the other one to tension. The top of the valence bands shows positive or negative $\Delta_{\mathrm{CF}}$ values, as expected.

The energy-splitting parameter $\Delta_{\mathrm{CF}}$ can actually be considered as the performance indicator in chalcopyrites including both the simple ternary and pseudocubic structures. It also acts as the bridge, linking the performance to the structural design, as will be discussed. For tetragonal chalcopyrites, either $\Gamma_{4 v}$ or $\Gamma_{5 v}$ bands (Figure S1) dominate p-type electrical transport. Usually the non-degenerate $\Gamma_{4 \mathrm{v}}$ is a light-hole band and the doubly degenerate $\Gamma_{5 \mathrm{v}}$ is a heavy-hole band. As already noted, for the optimal power factor it is desirable that $\Gamma_{4 \mathrm{v}}$ and $\Gamma_{5 \mathrm{v}}$ are nearly degenerate bands. ${ }^{[2,4,25,26]}$ By using the Boltzmann transport theory, the power factors and their dependence on $\Delta_{\mathrm{CF}}$ are calculated for a few 


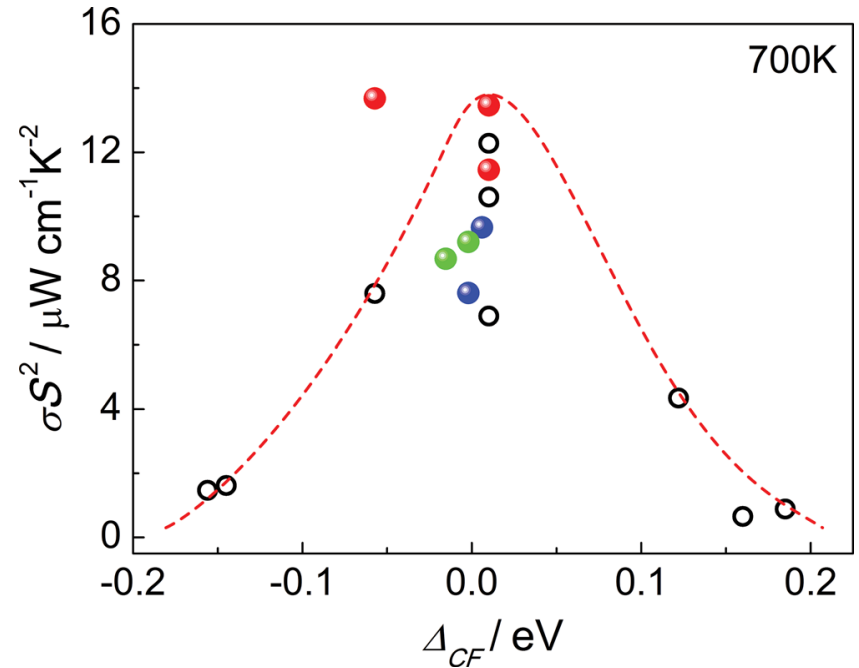

Figure 2. Thermoelectric power factors ${ }^{[14-22]}\left(\sigma S^{2}\right)$ at $700 \mathrm{~K}$ and their correlation with the crystal field splitting $\Delta_{\mathrm{CF}}$ in tetragonal chalcopyrites. Reported chalcopyrite compounds with $z T$ values above unity ${ }^{[14,17]}$ at high temperature are marked in red. The blue and green points are for $(\mathrm{Ag}, \mathrm{Cu})$ $\mathrm{InTe}_{2}$ and $\mathrm{Cu}(\mathrm{In}, \mathrm{Ga}) \mathrm{Te}_{2}$ mixtures or solutions, respectively.

typical chalcopyrites (see Figure S5, Supporting Information). The results confirm that the power factors show peak values when $\Delta_{\mathrm{CF}}$ approaches zero, consistent with the above discussion. Figure 2 plots the correlation between the measured power factors and $\Delta_{\mathrm{CF}}$ for all reported TE chalcopyrites, ${ }^{[14-22]}$ including two samples from the current work. The trend clearly demonstrates that the peak power factors are achieved around $\Delta_{\mathrm{CF}}=0$. It is worthwhile to note that the trend in Figure 2 is fully consistent with the reported $z T$ dependence on $\Delta_{\mathrm{CF}}$ shown in Figure 1c and Figure S10 in the Supporting Information. Different from PbTe ${ }^{[4]}$, the similar $z T$ variation trends vs. changing temperatures (Figure $1 \mathrm{c}$ and Figure S10) indicate that the band splitting $\Delta_{\mathrm{CF}}$ is almost not changed when shifting temperature, probably due to the fact that $\Gamma_{4 \mathrm{~V}}$ and $\Gamma_{5 \mathrm{~V}}$ bands of chalcopyrite compounds are located at the same symmetry point of Brillouin zone. Values of $z T$ increase significantly as $\left|\Delta_{\mathrm{CF}}\right|$ approaches zero. In a few systems, mainly those with nearly zero $\Delta_{\mathrm{CF}}$, the reported maximum $z T$ values reach about $1.2-1.4$ at high temperatures, ${ }^{[14,17]}$ comparable to other state-ofthe-art TE materials.

Deviations in tetragonal chalcopyrite from the cubic zinc blende structure are conveniently described by two parameters $u$ and $\eta$, where $u$ is the anion displacement from the regular center position $(1 / 4,1 / 4,1 / 4)$ in deformed tetrahedra and $\eta$ $(=c / 2 a)$ is the tetragonal distortion parameter representing compression $(<1)$ or tension $(>1)$ in the crystal lattice along the $z$-axis. ${ }^{[27,28]}$ Using density functional theory (DFT) calculations (see Supporting Information), the effect of parameters $u$ and $\eta$ on $\Delta_{\mathrm{CF}}$ is analyzed. As shown in Figure $3, \Delta_{\mathrm{CF}}$ changes little $( \pm 0.04 \mathrm{eV}$ at most) as a result of $10 \%$ variations in $u$ around the equilibrium positions of three representative tetragonal chalcopyrites. In contrast, $\Delta_{\mathrm{CF}}$ changes can reach $\pm(0.18-0.49) \mathrm{eV}$ when $\eta$ varies $10 \%$, and a strong linear dependence of $\Delta_{\mathrm{CF}}$ on the parameter $\eta$ is shown in Figure $3 \mathrm{~b}$. Therefore, it is reasonable to conclude that $\Delta_{\mathrm{CF}}$ correlates strongly with $\eta$ in tetragonal
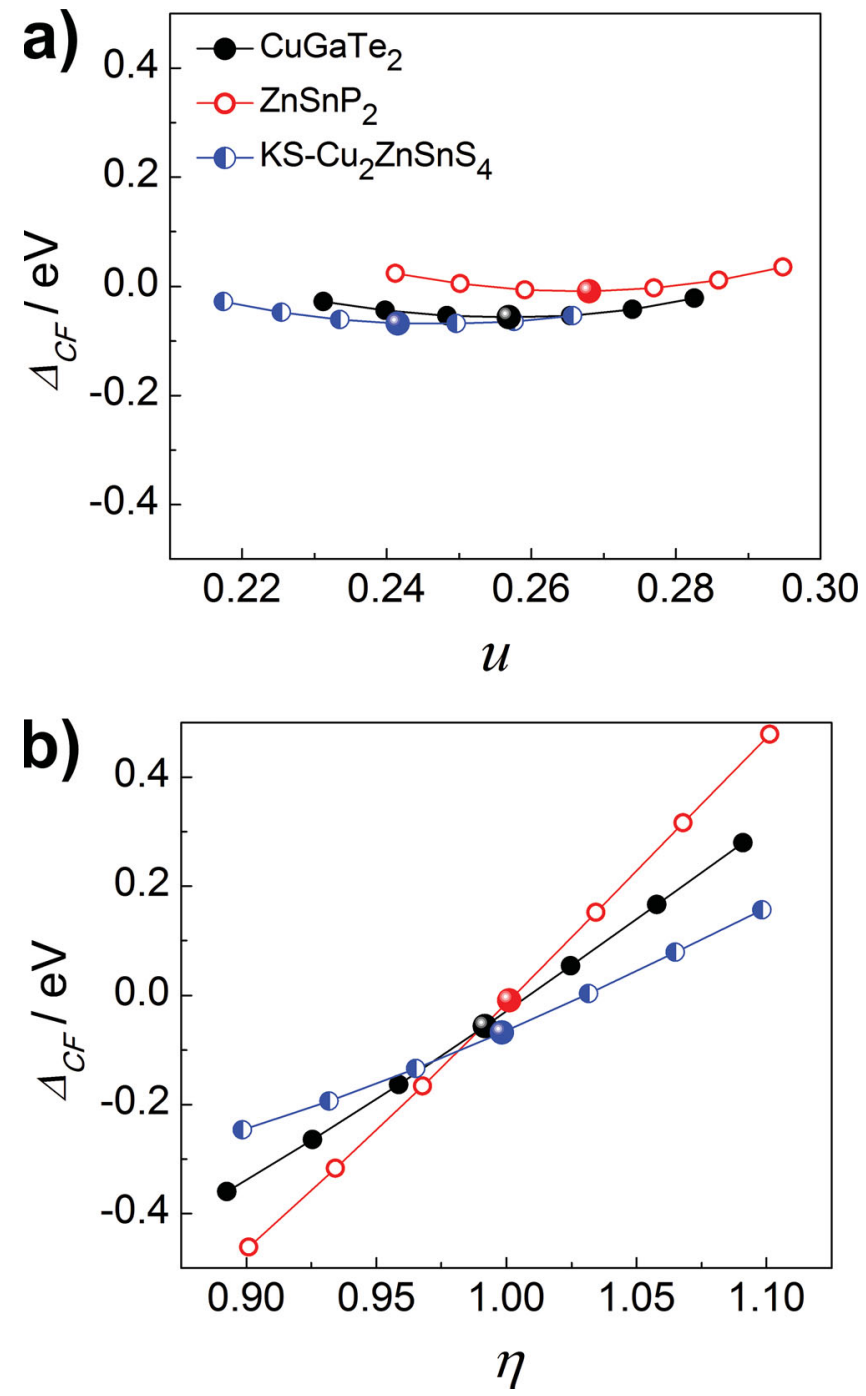

Figure 3. Parameters affecting $\Delta_{\mathrm{CF}}$ in three representative tetragonal chalcopyrite compounds I-III-VI $\mathrm{V}_{2}\left(\mathrm{CuGaTe}_{2}\right), \mathrm{II}-\mathrm{IV}-\mathrm{V}_{2}\left(\mathrm{ZnSnP}_{2}\right)$, and KS-I $-\mathrm{II}-$ $\mathrm{IV}-\mathrm{VI}_{4}\left(\mathrm{Cu}_{2} \mathrm{ZnSnS}_{4}\right)$. a) parameter $u$. b) parameter $\eta$. KS means kesterite structure. The relatively enlarged points represent equilibrium positions. $u$ and $\eta$ vary $10 \%$ around their equilibrium positions.

chalcopyrites, that is, the compression or tension stress along the $z$-axis (parameter $\eta$ ), ${ }^{[23,29]}$ instead of local anion displacements, govern the magnitude of the valence band split $\Delta_{\mathrm{CF}}$.

With the above-established relations among electronic transport parameters, $z$ T's, $\Delta_{\mathrm{CF}}$, and the distortion parameter $\eta$, it is possible to evaluate and quantify the TE performance of a large number of chalcopyrite semiconductors. Figure 4 depicts the calculated $\Delta_{\mathrm{CF}}$ as a function of $\eta$ for tetragonal chalcopyrites. For brevity, only a few typical systems are shown. A nice linear correlation is observed, consistent with the quasicubic model proposed by Hopfield. ${ }^{[30]}$ Thus, the relation between $\Delta_{\mathrm{CF}}$ and $\eta$ can be described by $\Delta_{\mathrm{CF}}=3 b(1-\eta)$, ${ }^{[29]}$ where $b$ is a parameter relevant to the structure distortion and equals to $-1.05 \mathrm{eV}$ in I-III-VI ${ }_{2}$, II-IV-V ${ }_{2}$, and KS-I $-\mathrm{II}^{-I V-V I} \mathrm{I}_{4}$ tetragonal chalcopyrite compounds by fitting the points shown in Figure 4. 


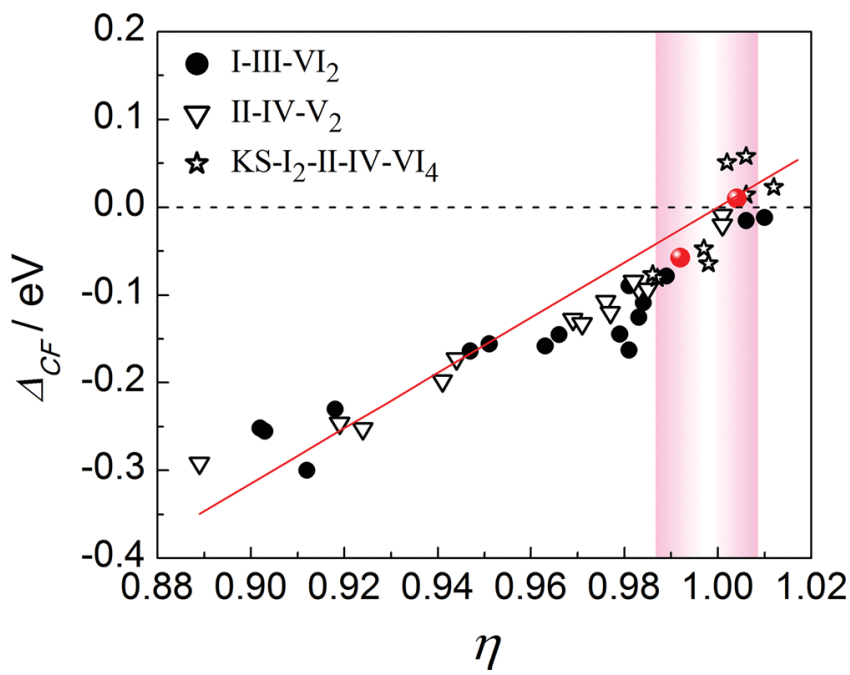

Figure 4. Calculated $\Delta_{\mathrm{CF}}$ Vs. $\eta$ correlation in I-III-VI, $\mathrm{II}-\mathrm{IV}-\mathrm{V}_{2}$ and $\mathrm{KS}-\mathrm{I}_{2}-\mathrm{II}-$ $\mathrm{IV}-\mathrm{VI}_{4}$ tetragonal chalcopyrites. KS means kesterite structure. Reported chalcopyrite compounds with $z T$ values above unity ${ }^{[14,17]}$ at high temperature are marked in red.

Figure 4 suggests that values of $\Delta_{\mathrm{CF}} \approx 0$ correspond to an ideal value of $\eta \approx 1$. The above results present a simple but insightful picture that TE transport properties should show the same trend as a function of $\eta$ as they do as a function of $\Delta_{\mathrm{CF}}$, displayed in Figure S5-S6 in the Supporting Information. Hence, to be potentially good TE materials, chalcopyrites should have a tetragonal distortion parameter around unity (we could call it the unity- $\eta$ rule). When $\eta$ is around 1 , the power factors display peak values in complete agreement with the pseudocubic picture of cubic-like degenerate electronic bands yielding good electronic transport and thus high TE performance. Moreover, the quality factor $B$, assessing the thermoelectric performance of a material, is proportional to $\mu_{\mathrm{H}}\left(m^{*} / m_{\mathrm{e}}\right)^{3 / 2} / \kappa_{\mathrm{L}},{ }^{[3,31]}$ where $\mu_{\mathrm{H}}$ is the mobility of carriers, $m^{*}$ is the electronic effective mass, $m_{\mathrm{e}}$ is the mass of an electron, and $\kappa_{\mathrm{L}}$ is the lattice thermal conductivity. Values of $\kappa_{\mathrm{L}}$ in tetragonal chalcopyrites are usually low, typically less than $1 \mathrm{~W} \mathrm{~m}^{-1} \mathrm{~K}^{-1}$ at high temperatures, and very close to each other due to the large lattice distortion. Thus, the $z T$ is mainly determined by electrical properties, and the relative performance of a group of compounds can be evaluated by the parameter $\mu_{\mathrm{H}}\left(m^{*} / m_{\mathrm{e}}\right)^{3 / 2}$. Figure S7 in the Supporting Information shows $\mu_{\mathrm{H}}\left(m^{*} / m_{\mathrm{e}}\right)^{3 / 2}$ as a function of $\eta$ for many reported chalcopyrites. As expected, the curve also shows the same trend as the measured power factors, even using the acoustic phonon scattering and single parabolic band approximation to evaluate the Hall data. The similar trend for the quality factors (Figure S7) and the power factors (Figure 2) suggests that the parameter $u$ has only a weak effect on the carrier mobility in chalcopyrite compounds. This again confirms the conclusion of "unity- $\eta$ rule" for good thermoelectricity.

The unity- $\eta$ rule provides a systematic strategy for guiding the evaluation and optimization of TE chalcopyrites. Because the parameter $\eta$ can be easily obtained from lattice parameters $a$ and $c$, the unity- $\eta$ rule can be straightforwardly applied to the evaluation of TE performance of all tetragonal chalcopyrites.
The pink-colored band in Figure 4 marks the region of chalcopyrite compounds with $\eta$ around unity that are potentially good TE materials. The big advantage of the rule is its predictive power to dismiss from further considerations chalcopyrite compounds that show large deviations of the parameter $\eta$ from unity. On the other hand, the reported TE chalcopyrites with $z T$ above unity indeed fall within the pink-colored band shown in Figure 1c, validating this selection rule. Numerous potential good novel TE compounds based on the calculated $\eta$ values are listed in Table S1 in the Supporting Information. Here we use the selection criteria $0.987 \leq \eta \leq 1.008$ and bandgap $E_{\mathrm{g}}<1.2 \mathrm{eV}$ to search for prospective ternary and quaternary $\mathrm{TE}$ chalcopyrites. Compounds, such as $\mathrm{KS}-\mathrm{Cu}_{2} \mathrm{CdGeSe}_{4}, \mathrm{KS}$ $\mathrm{Cu}_{2} \mathrm{ZnSnSe}{ }_{4}$ and $\mathrm{KS}-\mathrm{Cu}_{2} \mathrm{CdSnSe}_{4}$ (KS: Kesterite Structure), are expected to possess good electronic transport properties as well as TE performance because of the near-ideal values of $\eta$ and suitable bandgaps. Actually, the unity- $\eta$ rule also clarifies the optimization strategy of chalcopyrites: for compounds with $\eta$ greater than unity, reducing $\eta$ is probably more effective, while for compounds with $\eta$ smaller than unity, the approach should focus on increasing $\eta$.

The unity- $\eta$ rule can also be extended to the design of novel pseudocubic multinary high-performance TE chalcopyrites. Figure 5a shows that the cubic-like cation framework can be maintained in solid solutions or mixed multinary chalcopyrites, although the simulation indicates that there are a variety of tetrahedra with arbitrary deformation and different anion displacements due to nearly random distribution of the anions. Similarly, cubic-like valence band edges are observed as a result of symmetry operations on the cubic framework in supercells (see Figure 5b). Taking $\mathrm{Cu}_{0.875} \mathrm{Ag}_{0.125} \mathrm{InTe}_{2}$ as an example, the valence bands at $\Gamma$ point are highly degenerate, showing an ideal character of pseudocubic structures as shown in Figure 1. Figure 5c maps the $\eta$ vs. a dependence for chalcopyrite compounds where $a$ is the lattice constant. Only a few systems, those with bandgaps $<1.7 \mathrm{eV}$, are shown out of a myriad of chalcopyrite compounds. The pseudocubic approach with the aid of the unity- $\eta$ rule and the $\eta$ - $a$ map, is very effective in screening prospective solid solutions and mixtures of chalcopyrite compounds. As the overall objective is to maintain an ideal $\eta=1$ value, one may select a few compounds with higher $(>1)$ and lower $(<1) \eta$ values and reasonable lattice mismatch and attempt to form a solid solution or mixed state with $\eta \approx 1$ by appropriately varying the molar ratio of the two constituent compounds.

Density functional calculations for a few selected solid solution systems, $\mathrm{CuIn}_{1-x} \mathrm{Ga}_{x} \mathrm{Te}_{2}, \quad \mathrm{Cu}_{1-x} \mathrm{Ag}_{x} \mathrm{InTe}_{2}$ and $\mathrm{KS}-\mathrm{Cu}_{2} \mathrm{Cd}_{x} \mathrm{Zn}_{1-x} \mathrm{GeSe}_{4}$ ( $x$ is solubility) shown by the red stars in Figure $5 c$, prove that the desired solid solution or mixed ratio is indeed possible to realize with the ideal $\eta$ value around 1 . The dependence of $\eta$ and $\Delta_{\mathrm{CF}}$ on molar ratios of constituent compounds is given in Supporting Information. It is gratifying that the predicted performance of $\mathrm{CuIn}_{1-x} \mathrm{Ga}_{x} \mathrm{Te}_{2}$ and $\mathrm{Cu}_{1-x} \mathrm{Ag}_{x} \mathrm{InTe}_{2}$ solid solutions or mixtures is confirmed by experiments including our own data and the literature values. Figure $5 \mathrm{~d}$ shows the temperature dependent $z T$ values. Solid solutions/ mixtures of composition $\mathrm{Cu}_{1-x} \mathrm{Ag}_{x} \mathrm{InTe}_{2}$ and $\mathrm{CuIn}_{1-x} \mathrm{Ga}_{x} \mathrm{Te}_{2}$ with ideal $\eta$ values around 1 show much enhanced $z T$ values than single chalcopyrite compounds, consistent with 
a)

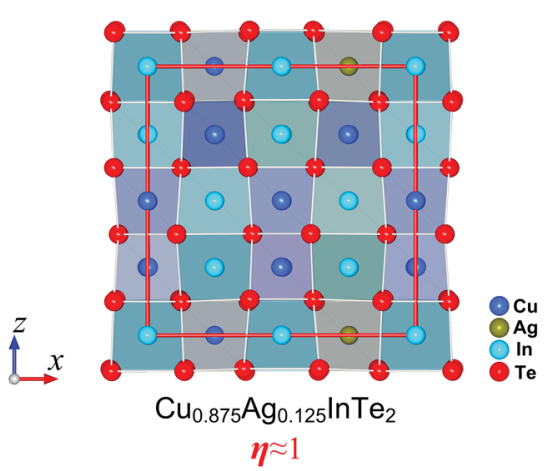

c)

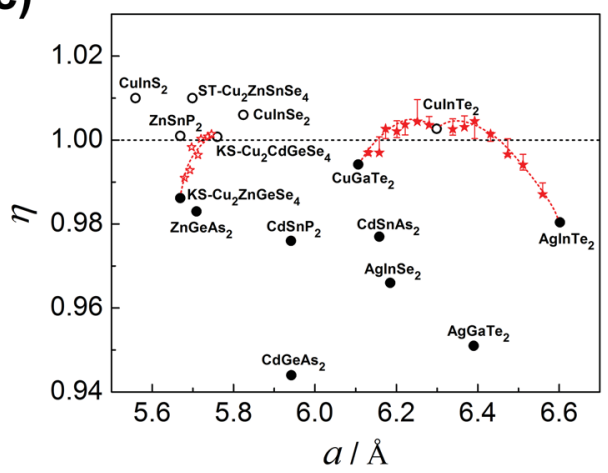

b)

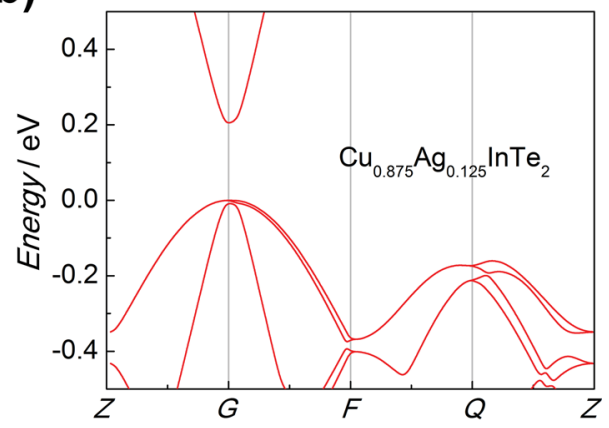

d)

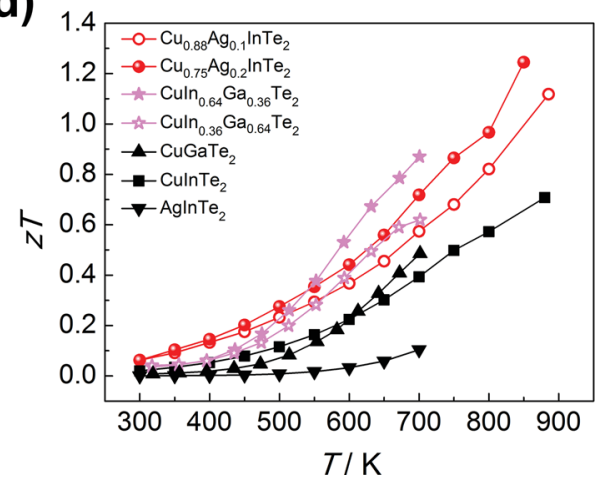

Figure 5. Crystal structure and electronic bands, $\eta$ vs. a compound map for designing high-performance multinary chalcopyrites, and temperature dependence of $z T$ for selected multinary chalcopyrite TE materials. a) Calculated projected plane representation of the crystal structure on the (010) plane for $\mathrm{Cu}_{0.875} \mathrm{Ag}_{0.125} \mathrm{InTe}_{2}$. The cation sublattice shows ideally cubic framework while the anion sublattice appears extremely distorted with anion atoms nearly randomly distributed. b) Electronic bands of $\mathrm{Cu}_{0.875} \mathrm{Ag}_{0.125} \mathrm{InTe}$. c) Calculated tetragonal distortion parameter $\eta$ as a function of the lattice constant $a$ in chalcopyrites with $E_{\mathrm{g}}<1.7 \mathrm{eV}$. The red stars with a trend line represent solid solutions and mixtures between two chalcopyrite compounds with higher $(>1)$ and lower $(<1) \eta$ values. The error bars are also given. ST and KS mean stannite structure and kesterite structure, respectively. d) Temperature dependent $z T$ values of $\mathrm{CuInTe}_{2}, \mathrm{CuGaTe}_{2}{ }^{[22]}\left(\mathrm{AgInTe}_{2}\right)$ and their solid solutions. Our own data for $(\mathrm{Cu}, \mathrm{Ag}) \operatorname{lnTe} e_{2}$ solutions are in red.

calculations and predictions. Details concerning TE properties of these ideal pseudocubic multinary chalcopyrites are shown in Supporting Information.

In this work, we demonstrate a new strategy to search for and design high-performance non-cubic TE materials through the utilization of a rational pseudocubic structure that supports cubic-like degenerate electronic bands, via the coexistence of long-range cubic framework with localized short-range non-cubic lattice distortions. We identify a simple yet powerful selection rule based on maintaining the distortion parameter $\eta$ near unity that is shown to be equivalent to minimizing the energy-splitting parameter $\Delta_{\mathrm{CF}}$. With the computed $\eta$ vs. lattice parameter maps, one can easily establish the correct molar ratio for two or more chalcopyrite compounds to form a solid solution with the desirable $\eta \approx 1$ value that assures excellent TE performance. The pseudocubic approach augmented by the unity- $\eta$ rule and the $\eta-a$ map, is a new paradigm that points a clear direction how to design high-performing tetragonal chalcopyrite-based TE materials. Using this approach, we predict a series of novel highly efficient chalcopyrite TE materials and, on a selected subset of them, we verify experimentally that they indeed possess significantly enhanced $z T$ values. The approach can be extended to other tetragonal semiconductors as well as other non-cubic materials with the aim to realize cubic-like degenerate electronic bands that support high TE performance.
This work thus addresses the interest of researchers to broaden the scope of prospective TE materials especially among noncubic semiconductors.

\section{Experimental Section}

First-Principles Calculations: Density functional theory calculations were carried out using the projector-augmented wave (PAW) method ${ }^{[32]}$ as implemented in the Vienna ab initio simulation package (VASP). ${ }^{[33]}$ The crystal structures of chalcopyrite semiconductors were relaxed using HSE06 functional. ${ }^{[34]}$ The plane-wave energy cutoff was set at $350 \mathrm{eV}$, and a $4 \times 4 \times 4$ Monkhorst-Pack $k$ mesh was used for crystal structure optimization. A $9 \times 9 \times 9$ Monkhorst-Pack $k$ mesh was used for electronic structure calculations. An energy convergence criterion of $10^{-4} \mathrm{eV}$ and a Hellmann-Feynman force convergence criterion of $0.008 \mathrm{eV} \AA^{-1}$ were adopted. Band-structure calculations were implemented by $\mathrm{PBE}+U$ approach, and $U=4 \mathrm{eV}^{[35,36]}$ was applied on $\mathrm{Cu} 3 \mathrm{~d}$ states, $\mathrm{Ag} 4 \mathrm{~d}$ states, $\mathrm{Zn} 3 \mathrm{~d}$ states, and $\mathrm{Cd} 4 \mathrm{~d}$ states. Details of electronic-structure calculations for solid solutions and that of the transport property modeling are given in the Supporting Information.

Experimental Details: Bulk polycrystalline $\mathrm{Cu}_{1-x} \mathrm{Ag}_{x} \operatorname{lnTe}_{2}$ were prepared by a melting and annealing method. Stoichiometric amounts of $\mathrm{Cu}$ (Alfa Aesar, 5N, shot), Ag (Alfa Aesar, 5N, granule), In (Alfa Aesar, $5 \mathrm{~N}$, granule), and Te (Alfa Aesar, $5 \mathrm{~N}$, shot) were loaded into graphite crucibles, and then sealed in evacuated silica tubes. The silica tubes were heated to $1100{ }^{\circ} \mathrm{C}$ and kept at this temperature for $12 \mathrm{~h}$, and then quenched in ice cold water and annealed at $650^{\circ} \mathrm{C}$ for $5 \mathrm{~d}$. Finally, the 
obtained ingots were crushed into a fine powder, followed by spark plasma sintering under a pressure of $65 \mathrm{MPa}$ at $550{ }^{\circ} \mathrm{C}$ for $10 \mathrm{~min}$, yielding densified bulk samples. High-temperature thermopower and electrical resistivity were measured using an Ulvac ZEM-3 system under a sealed chamber with a small amount of helium gas. The thermal diffusivity $(D)$ and heat capacity $\left(C_{P}\right)$ from $300 \mathrm{~K}$ to $1000 \mathrm{~K}$ were measured using the laser flash method (Netzsch, LFA427) and differential scanning calorimetry (Netzsch DSC 404F3), respectively. The density $(d)$ was measured using the Archimedes method. The thermal conductivity was calculated by $\kappa=D \times C_{p} \times d$. Hall effect measurements were performed using a cryostat equipped with a $5.5 \mathrm{~T}$ magnet.

\section{Supporting Information}

Supporting Information is available from the Wiley Online Library or from the author.

\section{Acknowledgements}

This work was supported by National Basic Research Program of China (973-program) under Project No. 2013CB632501, the National Natural Science Foundation of China (NSFC) under No. 11234012, 51121064, and 51222209. The Hall effect measurements were carried out at the University of Michigan and were supported by the Center for Solar and Thermal Energy Conversion, an Energy Frontier Research Center funded by the U.S. Department of Energy, Office of Science, Office of Basic Energy Sciences under Award No. DE-SC0000957.

Received: January 5, 2014

Revised: January 27, 2014

Published online: April 1, 2014

[1] J. Yang, H.-L. Yip, A. K. Y. Jen, Adv. Energy Mater. 2013, 3, 549.

[2] G. J. Snyder, E. S. Toberer, Nat. Mater. 2008, 7, 105.

[3] G. S. Nolas, J. Sharp, H. J. Goldsmid, in Thermoelectrics: Basic Principles and New Materials Developments, (Eds: A. Zunger, R. M. Osgood Jr., R. Hull, H. Sakaki), Springer Verlag, New York 2001, Ch. 3

[4] Y. Pei, X. Shi, A. LaLonde, H. Wang, L. Chen, G. J. Snyder, Nature 2011, 473, 66

[5] H. L. Liu, X. Shi, F. F. Xu, L. L. Zhang, W. Q. Zhang, L. D. Chen, Q. Li, C. Uher, T. Day, G. J. Snyder, Nat. Mater. 2012, 11, 422.

[6] A. I. Hochbaum, R. Chen, R. D. Delgado, W. Liang, E. C. Garnett, M. Najarian, A. Majumdar, P. Yang, Nature 2008, 451, 163.

[7] J. P. Heremans, V. Jovovic, E. S. Toberer, A. Saramat, K. Kurosaki, A. Charoenphakdee, S. Yamanaka, G. J. Snyder, Science 2008, 321, 554

[8] B. C. Sales, D. Mandrus, R. K. Williams, Science 1996, 272, 1325.
[9] K. Biswas, J. He, I. D. Blum, C.-I. Wu, T. P. Hogan, D. N. Seidman, V. P. Dravid, M. G. Kanatzidis, Nature 2012, 489, 414.

[10] K. F. Hsu, S. Loo, F. Guo, W. Chen, J. S. Dyck, C. Uher, T. Hogan, E. K. Polychroniadis, M. G. Kanatzidis, Science 2004, 303, 818.

[11] K. Biswas, J. He, Q. Zhang, G. Wang, C. Uher, V. P. Dravid, M. G. Kanatzidis, Nat. Chem. 2011, 3, 160.

[12] C. Uher, J. Yang, S. Hu, D. T. Morelli, G. P. Meisner, Phys. Rev. B $1999,59,8615$.

[13] V. K. Zaitsev, M. I. Fedorov, E. A. Gurieva, I. S. Eremin, P. P. Konstantinov, A. Y. Samunin, M. V. Vedernikov, Phys. Rev. B 2006, 74, 045207.

[14] T. Plirdpring, K. Kurosaki, A. Kosuga, T. Day, S. Firdosy, V. Ravi, G. J. Snyder, A. Harnwunggmoung, T. Sugahara, Y. Ohishi, H. Muta, S. Yamanaka, Adv. Mater. 2012, 24, 3622.

[15] M. Ibanez, R. Zamani, A. LaLonde, D. Cadavid, W. Li, A. Shavel, J. Arbiol, J. R. Morante, S. Gorsse, G. J. Snyder, A. Cabot, J. Am. Chem. Soc. 2012, 134, 4060.

[16] A. Kosuga, T. Plirdpring, R. Higashine, M. Matsuzawa, K. Kurosaki, S. Yamanaka, Appl. Phys. Lett. 2012, 100, 042108.

[17] R. Liu, L. Xi, H. Liu, X. Shi, W. Zhang, L. Chen, Chem. Commun. 2012, 48, 3818

[18] P. Z. Ying, H. Zhou, Y. L. Gao, Y. Y. Li, Y. P. Li, X. L. Lian, J. L. Cui, Key Eng. Mater. 2012, 519, 188.

[19] A. Yusufu, K. Kurosaki, A. Kosuga, T. Sugahara, Y. Ohishi, H. Muta, S. Yamanaka, Appl. Phys. Lett. 2011, 99, 061902.

[20] X. Y. Shi, F. Q. Huang, M. L. Liu, L. D. Chen, Appl. Phys. Lett. 2009, 94, 122103

[21] M. L. Liu, F. Q. Huang, L. D. Chen, I. W. Chen, Appl. Phys. Lett. 2009, 94, 202103

[22] Y. Li, Q. Meng, Y. Deng, H. Zhou, Y. Gao, Y. Li, J. Yang, J. Cui, Appl. Phys. Lett. 2012, 100, 231903.

[23] J. Rowe, J. Shay, Phys. Rev. B 1971, 3, 451.

[24] L. Artus, Y. Bertrand, C. Ance, J. Phys. C: Solid State Phys. 1986, 19, 5937.

[25] W. Liu, X. Tan, K. Yin, H. Liu, X. Tang, J. Shi, Q. Zhang, C. Uher, Phys. Rev. Lett. 2012, 108, 166601.

[26] X. Liu, T. Zhu, H. Wang, L. Hu, H. Xie, G. Jiang, G. J. Snyder X. Zhao, Adv. Energy Mater. 2013, 3, 1238.

[27] J. Martins, A. Zunger, Phys. Rev. B 1985, 32, 2689.

[28] J. Jaffe, A. Zunger, Phys. Rev. B 1984, 29, 1882.

[29] J. L. Shay, J. H. Wernick, Ternary Chalcopyrite Semiconductors: Growth, Electronic Properties and Applications, Pergamon, Oxford, UK, 1975

[30] J. J. Hopfield, J. Phys. Chem. Solids 1960, 15, 97.

[31] Y. Pei, H. Wang, G. J. Snyder, Adv. Mater. 2012, 24, 6125.

[32] P. E. Blöchl, Phys. Rev. B 1994, 50, 17953.

[33] G. Kresse, J. Furthmüller, Phys. Rev. B 1996, 54, 11169.

[34] J. Paier, M. Marsman, K. Hummer, G. Kresse, I. C. Gerber, J. G. Angyan, J. Chem. Phys. 2006, 124, 154709.

[35] Y. Zhang, X. Yuan, X. Sun, B.-C. Shih, P. Zhang, W. Zhang, Phys. Rev. B 2011, 84, 075127

[36] Y. Zhang, J. Zhang, W. Gao, T. A. Abtew, Y. Wang, P. Zhang, W. Zhang, J. Chem. Phys. 2013, 139, 184706. 